\title{
NOTA
}

\section{ABSORÇÃO, TRANSLOCAÇÃO E UTILIZAÇÃO DE FÓSFORO POR VARIEDADES DE TRIGO SUBMETIDAS À TOXIDEZ DE ALUMÍNIO(1)}

\author{
A. D. ABICHEQUER ${ }^{(2)}, H^{\text {B BOHNEN }}{ }^{(3)} \&$ I. ANGHINONI (4)
}

\begin{abstract}
RESUMO
Variedades de trigo tolerantes ao alumínio podem ser mais eficientes no aproveitamento de fósforo na presença desse elemento. $\mathbf{O}$ objetivo deste trabal ho foi avaliar a influência do alumínio em variedades de trigo Toropi, considerada eficiente, e CNT 8, ineficiente quanto ao aproveitamento de fósforo. Realizou-se um experimento em 1994, em casa de vegetação, em vasos que conti nham solução nutritiva com duas concentrações de fósforo $\left(1,6\right.$ e $\left.32,3 \mu \mathrm{mol} \mathrm{L}^{-1}\right)$ e duas de

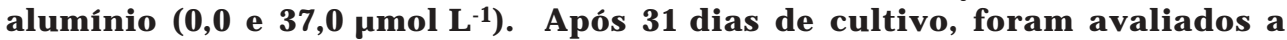
morfologia das raízes e o aproveitamento do fósforo. As raízes da variedade de trigo Toropi foram menos afetadas do que as da CNT 8 pelo alumínio; no entanto, não houve relação da absorção de fósforo com o comprimento, raio médio e superfície do sistema radicular. A presença de alumínio aumentou a diferença entre as variedades Toropi e CNT 8 quanto à eficiência de absorção, translocação e utilização de fósforo, com vantagem para a primeira, que é mais tolerante ao alumínio.
\end{abstract}

Termos de indexação: eficiência, nutrição vegetal, aproveitamento de nutrientes, genótipos, tolerância.

\section{SUMMARY: PHOSPHORUS UPTAKE, TRANSLOCATION AND UTILIZATION IN WHEAT VARIETIES UNDER ALUMINUM TOXICITY}

Aluminum tolerant wheat varieties can present increased efficiency of phosphorus use in presence of aluminum. A greenhouse experiment was conducted in 1994 to compare the wheat cultivars (Toropi) and (CNT 8), considered efficient and inefficient, respectively, in

\footnotetext{
(1) Parte da Tese de Mestrado do primeiro autor, apresentada à Faculdade de Agronomia da Universidade Federal do Rio Grande do Sul - UFRGS. Recebido para publicação em dezembro de 1999 e aprovado em fevereiro de 2003.

(2) Pesquisador da Fundação Estadual de Pesquisa Agropecuária - FEPAGRO. Rua Gonçalves Dias 570, CEP 90130-060 Porto Alegre (RS). E-mail: andredab@bewnet.com.br

(3) Engenheiro-Agrônomo, Colaborador Convidado do Departamento de Solos da Faculdade de Agronomia da Universidade Federal do Rio Grande do Sul - UFRGS. Caixa Postal 776, CEP 90001-970 Porto Alegre (RS). E-mail: bohnen@conex.com.br

(4) Professor do Departamento de Solos da Faculdade de Agronomia da Universidade Federal do Rio Grande do Sul - UFRGS. Caixa Postal 776, CEP 90001-970 Porto Alegre (RS). E-mail: ibanghi@vortex.ufrgs.br
} 
relation to phosphorus use. Nutrient solutions containing two phosphorus (1.6 and $\left.32.3 \mu \mathrm{mol} \mathrm{L}^{-1}\right)$ and two al umi num $\left(0.0\right.$ and $\left.37.0 \mu \mathrm{mol} \mathrm{L}^{-1}\right)$ concentrations weretested. Root morphology and phosphateuse were established for the plants, which wereharvested from the pots on the $31^{\text {th }}$ day. Roots of the Toropi cultivar were less affected by aluminum than CNT 8; there was no relationship, however, between phosphorus uptake and root length, radius or surface. The presence of aluminum increased the difference between Toropi and CNT 8 in their efficiency of phosphorus uptake, transl ocation and utilization. Toropi, which is more tol erant towards alumi num, proved to be more efficient.

Index terms: efficiency, plant nutrition, nutrient use, genotypes, tol erance

\section{INTRODUÇÃO}

Diferenças entre variedades de trigo quanto à eficiência no aproveitamento ou uso de fósforo podem estar relacionadas com o diferentegrau detol erância dessas variedades à toxidez dealumínio, comum nos solos ondeo cereal écultivado no Brasil. A eficiência no aproveitamento de fósforo resulta da eficiência de absorção, de transl ocação e de utilização (Baligar \& Fageria, 1999).

O alumínio prejudica a absorção de fósforo de duas formas: reduz o crescimento do sistema radicular ( $F$ oy, 1976) e liga-se ao fósforo, tanto na superfície quanto no interior das células, formando compostos de baixa solubilidade (Clarkson, 1966). Desta forma, ocorre redução na absorção de fósforo, bem como precipitação do nutriente nas raízes, restringindo a sua translocação para a parte aérea.

Variedades de trigo tolerantes ao alumínio são menos prejudicadas do que as sensíveis (Miranda, 1985), especialmente em sol os ácidos e com baixa disponibilidade de fósforo, como é o caso de solos tropicais esubtropicais. Cultivares desoja tolerantes ao alumínio foram menos afetadas por essa toxidez, tanto na morfologia das raízes quanto na cinética de absorção de fósforo (Vilela \& Anghinoni, 1984). Cultivares de trigo tolerantes ao alumínio foram, também, mais eficientes na translocação de fósforo em sol os com problemas detoxi dez daquel eel emento, sobretudo em cultivares mais sensíveis (Miranda, 1985; Alves et al., 1988).

Entretanto, nem semprea eficiência de absorção e a utilização de fósforo estão relacionadas diretamente com a tolerância ao alumínio. Assim, a variedade de trigo $\mathrm{BH}$ 1146, mesmo sendo uma das fontes de maior tolerância ao alumínio utilizada universal menteem programas de melhoramento de trigo, apresentou menor absorção de fósforo do que outros genótipos (Soon, 1992). Da mesma forma, não houve relação entre índices de eficiência de aproveitamento de fósforo e a tolerância de linhagens de milhoe arroz aoalumínio (Furlani \& Furlani, 1991).

A tolerância ao alumínio, classicamente, é explicada por dois grupos de mecanismos: mecanismos de exclusão, que impedem que o alumínio alcance seus síti os detoxidez na planta; mecanismos internos (de reparo), que possibilitam a penetração do alumínio no interior da célula, mas têm sua ação fitotóxica neutralizada (Cançado et al., 1999). Diversos mecanismos bioquímicos têm sido propostos para explicar a tolerância.

No caso da exclusão, podem-se citar: a exsudação radicular de moléculas quelantes (principalmente ácidos orgânicos) que complexam o alumínio, a elevação do $\mathrm{pH}$ da rizosfera pelas raízes, a baixa CTC das raízes, a síntese de mucilagem no ápice radicular esíntese exsudação de polipeptídeos. No caso da tolerância interna, são citadas: a ação de polipeptídeos do citoplasma como moléculas quelantes, a existência de enzimas cuja atividade não é prejudicada pelo alumínio e a eliminação do alumínio do ambiente celular por compartimentalização no vacúolo.

Quanto à herança genética da tolerância ao alumínio, considera-se, na maioria das espécies vegetais, que é característica dominante e poligênica, podendo ser controlada por um ou mais genes de ação maior e diversos genes modificadores (Cançado et al., 1999).

O trabal hotevecomo objetivo avaliar a influência do alumínio na morfol ogia das raízes ena absorção, transl ocação e utilização de fósforo por variedades de trigo com diferentes graus de aproveitamento deste nutriente.

\section{MATERIAL E MÉTODOS}

Foram utilizadas as variedades de trigo (Triticum vulgare) Toropi, considerada eficiente, e CNT 8, ineficiente quanto ao aproveitamento de fósforo, de acordo com A bichequer \& Bohnen (1998). A Toropi é, também, considerada tolerante e a CNT 8, sensível ao alumínio, conforme demonstrado por Camargo et al. (1987).

O trabal ho foi realizado nos meses de setembro e outubro de 1994, em casa de vegetação do Departamento de Solos da U niversidade F ederal do Rio Grande do Sul, em Porto Alegre (RS). As duas variedades foram submetidas a duas concentrações defósforo (1,6 e 32,3 $\left.\mu \mathrm{mol} \mathrm{L}^{-1}\right)$ ea duas concentrações 
de alumínio $\left(0,0\right.$ e $\left.37,0 \mu \mathrm{mol} \mathrm{L}^{-1}\right)$ em solução nutritiva, com os vasos arranjados no del ineamento compl etamente casualizado, com três repetições. As concentrações de fósforo e alumínio foram estabel ecidas em experimento preliminar. O alumínio foi adicionado como $\mathrm{Al}\left(\mathrm{SO}_{4}\right)_{3} \cdot 18 \mathrm{H}_{2} \mathrm{O}$ e o fósforo como $\mathrm{KH}_{2} \mathrm{PO}_{4}$. A composição do restante da solução nutritiva foi a mesma utilizada por Abichequer \& Bohnen (1998) e constou de: 2,0 mmol L-1 de $\mathrm{K}(\mathrm{KCl}) ; 1,0 \mathrm{mmol}^{-1}$ de $\mathrm{Mg}$ e de $\mathrm{S}$ $\left(\mathrm{MgSO}_{4} .7 \mathrm{H}_{2} \mathrm{O}\right) ; 4,0 \mathrm{mmol}^{-1}$ de N e 1,5 mmol L-1 de $\mathrm{Ca}\left[\mathrm{NH}_{4} \mathrm{NO}_{3}, \mathrm{Ca}\left(\mathrm{NO}_{3}\right)_{2} \cdot 4 \mathrm{H}_{2} \mathrm{O}\right.$ e $\mathrm{CaCl}_{2} .2 \mathrm{H}_{2} \mathrm{O}$ ]; $3,0 \mathrm{mmol} \mathrm{L}-1$ de Cl $\left(\mathrm{KCl}^{-} \mathrm{CaCl}_{2} .2 \mathrm{H}_{2} \mathrm{O}\right) ; 105 \mu \mathrm{mol} \mathrm{L}^{-1}$ deFe(Fe-EDTA); 4,5 $\mu \mathrm{mol} \mathrm{L-1}$ deMn $\left(\mathrm{MnSO}_{4} \cdot \mathrm{H}_{2} \mathrm{O}\right)$; $4,7 \mu \mathrm{mol} \mathrm{L}^{-1}$ de $\mathrm{Cu}\left(\mathrm{CuSO}_{4} \cdot 5 \mathrm{H}_{2} \mathrm{O}\right) ; 1,5 \mu \mathrm{mol} \mathrm{L}-1$ de $\mathrm{Zn}\left(\mathrm{ZnSO}_{4} \cdot 7 \mathrm{H}_{2} \mathrm{O}\right) ; 23,1 \mu \mathrm{mol} \mathrm{L}-1$ de $\mathrm{B}\left(\mathrm{H}_{3} \mathrm{BO}_{3}\right) \mathrm{e}$ $0,1 \mu \mathrm{mol} \mathrm{L}-1$ de $\mathrm{Mo}\left(\mathrm{Na}_{2} \mathrm{MoO}_{4} \cdot 2 \mathrm{H}_{2} \mathrm{O}\right)$.

As sementes das variedades de trigo foram colocadas para germinar em papel filtro que continha solução de $0,5 \mathrm{mmol} \mathrm{L}^{-1}$ de $\mathrm{Ca}\left(\mathrm{NO}_{3}\right)_{2} \cdot 2 \mathrm{H}_{2} \mathrm{O}$. Aos cinco dias, quatro plântulas homogêneas foram transplantadas para vasos de dois litros, sendo fixadas em tampas de isopor. $\mathrm{O}$ pH da solução nutritiva foi mantidoem $4,1 \pm 0,2$ ea solução arejada continuamente, com troca a cada dois dias.

A col heita do experimento ocorreu após 31 dias de cultivo, quando as plantas estavam com 36 dias de idade. A parte aérea eas raízes foram avaliadas separadamente para obtenção do rendimento de matéria seca. Nas raízes, foram avaliados o comprimento, pelo método de Tennant (1975), e o raio e a superfície, por meio de fórmulas (Shenk \& Barber, 1979). O crescimento das raízes foi considerado como exponencial e a taxa calculada conforme Shenk \& Barber (1979).

O teor defósforo no tecido vegetal foi determinado após combustão, por seis horas, em mufla a $500^{\circ} \mathrm{C}$, pela utilização do método de Murphy \& Riley (1962), após dissolução das cinzas em $\mathrm{HNO}_{3} 2,0 \mathrm{~mol} \mathrm{~L}^{-1}$. Calculou-se ofósforo transl ocado da seguinteforma: fósforo translocado = (conteúdo de fósforo na parte aérea/conteúdo de fósforo na planta) x 100 . A eficiência deutilização defósforo foi calculada pelas relações propostas por Siddiqi \& Glass (1981).

A análise da variância dos resultados foi seguida pelo teste $F(P<0,05)$, para a discriminação das diferenças entre as médias. Também foi analisada a correlação entre o conteúdo de fósforo na planta e as características morfológicas das raízes.

\section{RESULTADOS E DISCUSSÃO}

\section{Absorção de fósforo e morfologia das raízes}

O conteúdo de fósforo nas plantas de trigo (Quadro 1) foi afetado $(P<0,05)$ tanto pelas variedades quanto pelas concentrações de fósforo e alumínio na solução (interação tríplice). Uma maior absorção desse nutriente foi constatada na Toropi no maior nível de fósforo na solução, tanto na presença quanto na ausência do alumínio. A presença deste elemento prejudicou a absorção de fósforo por ambas as variedades, mas a CNT 8 foi mais afetada. Rosa \& Camargo (1990) também observaram mai or absorção de fósforo pel a variedade Toropi, comparativamente à CNT 8, em solo ácido com alumínio em nível tóxico. Assim, o caráter de tolerância ao alumínio, apresentado pela Toropi, afetou, também, a eficiência da absorção de fósforo em relação à variedade mais sensível, o que foi comprovado por Miranda (1985).

Para as características morfológicas de raízes, ocorreu interação significativa apenas entre variedades e concentrações de alumínio na solução (Quadro 2). Os resultados evidenciaram uma maior tolerância da Toropi ao alumínio, uma vez que, apesar de a CNT 8 apresentar características morfológicas mais favoráveis à absorção de fósforo (mai or comprimento, área superfícial, taxa de crescimento e menor raio de raízes) na ausência do alumínio, tais características, à exceção do raio médio, não se diferenciaram na presença de alumínio. Nesta situação, ocorreu um decréscimo muito mais acentuado dos val ores das características morfológicas deraízes na CNT 8, quando comparados aos da Toropi.

A maior superfície de raízes apresentada pela CNT 8 na ausência de alumínio não resultou em

\section{Quadro 1. Conteúdo de fósforo total, na parte aérea e nas raízes de variedades de trigo submetidas a duas concentrações de fósforo, na ausência e presença de alumínio, em solução nutritiva}

\begin{tabular}{|c|c|c|c|c|}
\hline \multirow{4}{*}{ Variedade } & \multicolumn{4}{|c|}{ Concentração de Al ( $\left.\mu \mathrm{mol} \mathrm{L}^{-1}\right)$} \\
\hline & \multicolumn{2}{|c|}{0,0} & \multicolumn{2}{|c|}{37,0} \\
\hline & \multicolumn{4}{|c|}{ Concentração de P ( $\mu \mathrm{mol} \mathrm{L-1)}$} \\
\hline & 1,6 & 32,3 & 1,6 & 32,3 \\
\hline & \multicolumn{4}{|c|}{ P total, mg planta-1 } \\
\hline \multirow[t]{2}{*}{$\begin{array}{l}\text { Toropi } \\
\text { CNT } 8\end{array}$} & $\begin{array}{l}0,249 \mathrm{aB} \\
0,254 \mathrm{aB}\end{array}$ & $\begin{array}{l}3,685 \text { aA } \\
3,076 \text { bA }\end{array}$ & $\begin{array}{l}0,210 \mathrm{aB} \\
0,198 \mathrm{aB}\end{array}$ & $\begin{array}{l}2,576 \mathrm{aA} \\
1,598 \mathrm{bA}\end{array}$ \\
\hline & \multicolumn{4}{|c|}{$\mathrm{P}$ na parte aérea, mg planta-1 } \\
\hline \multirow[t]{2}{*}{$\begin{array}{l}\text { Toropi } \\
\text { CNT } 8\end{array}$} & $\begin{array}{l}0,182 \mathrm{aB} \\
0,182 \mathrm{aB}\end{array}$ & $\begin{array}{l}3,473 \text { aA } \\
2,742 \text { bA }\end{array}$ & $\begin{array}{l}0,157 \mathrm{aB} \\
0,134 \mathrm{aB}\end{array}$ & $\begin{array}{l}2,355 \mathrm{aA} \\
1,005 \mathrm{bA}\end{array}$ \\
\hline & \multicolumn{4}{|c|}{ P nas raízes, mg planta-1 } \\
\hline $\begin{array}{l}\text { Toropi } \\
\text { CNT } 8\end{array}$ & $\begin{array}{l}0,067 \mathrm{aB} \\
0,072 \mathrm{aB}\end{array}$ & $\begin{array}{l}0,211 \mathrm{bA} \\
0,334 \mathrm{aA}\end{array}$ & $\begin{array}{l}0,053 \mathrm{aB} \\
0,063 \mathrm{aB}\end{array}$ & $\begin{array}{l}0,221 \mathrm{bA} \\
0,594 \mathrm{aA}\end{array}$ \\
\hline
\end{tabular}

Médias de variedades seguidas de mesma letra minúscula, dentro de cada concentração de fósforo e de alumínio, e médias de concentrações de fósforo, seguidas de mesma letra maiúscula, dentro de cada variedade e concentração de alumínio, não diferem pelo teste $F(P<0,05)$. 
Quadro 2. Morfologia do sistema radicular de variedades de trigo, na ausência e presença de alumínio, em solução nutritiva

\begin{tabular}{|c|c|c|}
\hline \multirow{2}{*}{ Variedade } & \multicolumn{2}{|c|}{ Concentração de Al ( $\left.\mu \mathrm{mol} L^{-1}\right)$} \\
\hline & 0,0 & 37,0 \\
\hline & \multicolumn{2}{|c|}{ Comprimento, $\mathrm{cm}$} \\
\hline \multirow{3}{*}{$\begin{array}{l}\text { Toropi } \\
\text { CNT } 8\end{array}$} & 842 bA & 663 aA \\
\hline & $1271 \mathrm{aA}$ & $446 \mathrm{aB}$ \\
\hline & \multicolumn{2}{|c|}{ Raio médio, mm } \\
\hline \multirow{3}{*}{$\begin{array}{l}\text { Toropi } \\
\text { CNT } 8\end{array}$} & 0,145 aA & $0,150 \mathrm{bA}$ \\
\hline & $0,135 \mathrm{bB}$ & 0,172 aA \\
\hline & \multicolumn{2}{|c|}{ Área superficial, $\mathrm{cm}^{2}$ planta-1 } \\
\hline \multirow{3}{*}{$\begin{array}{l}\text { Toropi } \\
\text { CNT } 8\end{array}$} & 76,5 bA & 63,0 aA \\
\hline & 106,6 aA & $48,1 \mathrm{aB}$ \\
\hline & \multicolumn{2}{|c|}{ Taxa de crescimento, $\times 10^{-6} \mathrm{~cm} \mathrm{~s}^{-1}$} \\
\hline Toropi & $1,78 \mathrm{bA}$ & $1,69 \mathrm{aB}$ \\
\hline CNT 8 & 2,15 aA & $1,76 \mathrm{aB}$ \\
\hline
\end{tabular}

Médias de variedades seguidas de mesma letra minúscula, dentro de cada concentração de alumínio, e médias de concentrações de alumínio, seguidas de mesma letra maiúscula, dentro de cada variedade, não diferem pelo teste $F(P<0,05)$.

maior absorção de fósforo, tendo si do superada pela Toropi no nível mais el evado desse nutriente na sol ução (Quadro 1). Assim, a absorção de fósforo não se correlacionou com o comprimento ( $r=0,59$; ns), com a área superficial ( $r=0,72 ; n s)$, com o raio ( $r=$ 0,03 ; ns) e com a taxa de crescimento de raízes ( $r=$ 0,46; ns). A ausência de relação entre absorção de fósforo e morfol ogia de raízes também foi verificada no experimento de Abichequer \& Bohnen (1998).

Pesquisas querelacionam a absorção defósforo com características morfológicas de raízes em solução nutritiva têm sido publicadas na literatura (Vilela \& Anghinoni, 1984; Miranda, 1985; Martinez et al., 1993). A aeração da sol ução di minui ou mesmo pode anular o efeito do mecanismo de difusão no suprimento de fósforo às raízes (Gerloff \& Gabelman, 1983).

Assim, antes de se tirar conclusões definitivas a respeito da relação entre a eficiência de absorção de fósforo e as características do sistema radicular de variedades de trigo com diferente sensibilidade ao al umínio, devem ser consideradas pesquisas em solo, com validação no campo. Diferenças entrevariedades da mesma espécie desenvolvidas no sol o podem ser causadas pela presença de pêlos radiculares ou micorrizas em solos deficientes em fósforo, fatores que não são rel evantes em solução nutritiva.

\section{Translocação de fósforo}

A eficiência de translocação de fósforo para a parte aérea do trigo (Quadro 3) foi, a exemplo da absorção, dependente da variedade e dos níveis de alumínio e de fósforo (interação tríplice). As variedades aumentaram a translocação de fósforo com o aumento de sua concentração na solução nutritiva, exceto no caso da CNT 8 na presença de alumínio. Na ausência dealumínio, a Toropi foi mais eficiente na transl ocação de fósforo do que a CNT 8 na mai or concentração do nutriente. J á, na presença de alumínio, a translocação de fósforo na Toropi foi maior nas duas concentrações deste nutriente $(P)$ na solução. Na verdade, a presença dealumínio não influiu na translocação de fósforo na Toropi, porém diminuiu na CNT 8 em ambas as condições de suprimento de fósforo. A eficiência de translocação de fósforo reflete a partição de fósforo nas raízes e parte aérea (Quadro 1).

A maior capacidade de translocação de fósforo da Toropi, em relação à CNT 8, foi observada por Abichequer \& Bohnen (1998). Ocorreu maior retenção de fósforo nas raízes em condições de deficiência destenutriente, priorizando o crescimento das raízes em relação à parte aérea, conforme observado em trigo (Miranda, 1985), em soja (Martinez et al., 1985) e em diversas espécies (L oneragam \& Asher, 1967).

A variedade Toropi mostrou-se mais toleranteao alumínio e eficiente ao uso de fósforo, em relação a outras variedades, inclusive à CNT 8 (Camargo et al., 1987; Ben, 1989; Abichequer \& Bohnen, 1998). A sua alta eficiência na translocação de fósforo na presença de alumínio (Quadro 3) deve refletir sua capacidade de neutralizar o alumínio na raiz, ou fora dela, uma vez que o conteúdo de fósforo na raiz não foi afetado pelo alumínio, em ambos os níveis de fósforo (Cançado et al., 1999). Ao contrário, na CNT 8, o conteúdo de fósforo na raiz aumentou com a presença de alumínio, especialmente na maior concentração de fósforo (Quadro 1).

Quadro 3. Fósforo translocado para a parte aérea (percentagem do fósforo absorvido presente na parte aérea) de variedades de trigo submetidas a duas concentrações de fósforo, na ausência e presença de alumínio, em solução nutritiva

\begin{tabular}{|c|c|c|c|c|}
\hline \multirow{4}{*}{ Variedade } & \multicolumn{4}{|c|}{ Concentração de Al ( $\left.\mu \mathrm{mol} L^{-1}\right)$} \\
\hline & \multicolumn{2}{|c|}{0,0} & \multicolumn{2}{|c|}{37,0} \\
\hline & \multicolumn{4}{|c|}{ Concentração de $P\left(\mu \mathrm{mol} L^{-1}\right)$} \\
\hline & 1,6 & 32,3 & 1,6 & 32,3 \\
\hline & & & 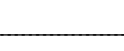 & \\
\hline $\begin{array}{l}\text { Toropi } \\
\text { CNT } 8\end{array}$ & $\begin{array}{l}73,3 \mathrm{aB} \\
71,6 \mathrm{aB}\end{array}$ & $\begin{array}{l}94,3 \text { aA } \\
89,2 \text { bA }\end{array}$ & $\begin{array}{l}74,8 \mathrm{aB} \\
67,9 \mathrm{bB}\end{array}$ & $\begin{array}{l}91,4 \mathrm{aA} \\
62,8 \mathrm{bA}\end{array}$ \\
\hline
\end{tabular}

Médias de variedades seguidas de mesma letra minúscula, dentro de cada concentração de fósforo e de alumínio, e médias de concentrações de fósforo, seguidas de mesma letra maiúscula, dentro de cada variedade e concentração de alumínio, não diferem pelo teste $F(P<0,05)$. 
Assim, a sensibilidade desta variedade ao alumínio deve estar relacionada com a falta de um mecanismo de sua neutralização, evidenciada pelo encurtamento e engrossamento das raízes (Quadro 2) e pelo acúmulo de fósforo nas raízes (Quadro 1).

Tal fato também foi verificado por Vilela \& Anghinoni (1984) em cultivares de soja, e, provavel mente, provocado pela formação de fosfato de alumínio (precipitado) e pela alta afinidade química entre os dois elementos. A avaliação da translocação de fósforo por variedades de trigo, por meio do exsudato xilemático (Alves et al., 1988), também mostrou redução de eficiência de translocação somentena mais sensível ena presença de alumínio, em concentração considerada tóxica.

Observa-se que, embora tenha havido redução na translocação de fósforo em variedades de trigo na presença de alumínio (Miranda, 1985), a variedade de trigo mais tolerante ao alumínio não foi mais eficientena translocação de fósforo do que a sensível. I sto significa que as características de tolerância ao alumínio e de eficiência na translocação do fósforo podem ocorrer associadas, pois a presença de alumínio e a deficiência de fósforo são comuns em solos ácidos, mas são comandadas por mecanismos e genes diferenciados (Cançado et al., 1999).

\section{Utilização de fósforo e produção de matéria seca}

A eficiência de utilização de fósforo (Quadro 4) foi influenciada pela interação de cultivares e concentrações de alumínio, não sendo significativas as demais interações. Desta forma, enquanto as variedades apresentaram eficiência de utilização semel hante na ausência de alumínio, tal eficiência diminuiu para ambas as variedades na presença de alumínio, porém com maior efeito sobre a CNT 8.

No caso da produção de matéria seca total e da parte aérea, ocorreu interação tríplice significativa. A Toropi apresentou maior produção dematéria seca total e da parte aérea (Quadro 5) do que a CNT 8

Quadro 4. Eficiência de utilização de fósforo de variedades de trigo, na ausência e presença de alumínio, em solução nutritiva

\begin{tabular}{ccc}
\hline \multirow{2}{*}{ Variedade } & \multicolumn{2}{c}{ Concentração de Al ( $\left.\boldsymbol{\mu m o l ~ L}^{-\mathbf{1}}\right)$} \\
\cline { 2 - 3 } & $\mathbf{0 , 0}$ & $\mathbf{3 7 , 0}$ \\
\hline & $-\mathrm{g}^{2}$ de $\mathrm{MS} \mathrm{mg}^{-1}$ de P \\
Toropi & $0,195 \mathrm{aA}$ & $0,170 \mathrm{aA}$ \\
CNT 8 & $0,203 \mathrm{aA}$ & $0,113 \mathrm{bB}$ \\
\hline
\end{tabular}

Médias de variedades seguidas de mesma letra minúscula, dentro de cada concentração de alumínio, e médias de concentrações de alumínio, seguidas de mesma letra maiúscula, dentro de cada variedade, não diferem pelo teste $F(P<0,05)$.
Quadro 5. Matéria seca total e da parte aérea de duas variedades de trigo submetidas a duas concentrações de fósforo, na ausência ou presença de alumínio, em solução nutritiva

\begin{tabular}{|c|c|c|c|c|}
\hline \multirow{4}{*}{ Variedade } & \multicolumn{4}{|c|}{ Concentração de Al ( $\left.\mu \mathrm{mol} L^{-1}\right)$} \\
\hline & \multicolumn{2}{|c|}{0,0} & \multicolumn{2}{|c|}{37,0} \\
\hline & \multicolumn{4}{|c|}{ Concentração de P ( $\left.\mu \mathrm{mol} L^{-1}\right)$} \\
\hline & 1,6 & 32,3 & 1,6 & 32,3 \\
\hline & & MS total, & planta-1 & \\
\hline \multirow[t]{2}{*}{$\begin{array}{l}\text { Toropi } \\
\text { CNT } 8\end{array}$} & $\begin{array}{l}0,248 a B \\
0,249 a B\end{array}$ & $\begin{array}{l}0,725 \text { aA } \\
0,708 \text { aA }\end{array}$ & $\begin{array}{l}0,210 \mathrm{aB} \\
0,176 \mathrm{aB}\end{array}$ & $\begin{array}{l}0,583 \text { aA } \\
0,331 \text { bA }\end{array}$ \\
\hline & \multicolumn{4}{|c|}{ MS da parte aérea, g planta-1 } \\
\hline $\begin{array}{l}\text { Toropi } \\
\text { CNT } 8\end{array}$ & $\begin{array}{l}0,173 \mathrm{aB} \\
0,151 \mathrm{aB}\end{array}$ & $\begin{array}{l}0,622 \mathrm{aA} \\
0,571 \mathrm{aA}\end{array}$ & $\begin{array}{l}0,150 \mathrm{aB} \\
0,125 \mathrm{aB}\end{array}$ & $\begin{array}{l}0,492 \mathrm{aA} \\
0,256 \mathrm{bA}\end{array}$ \\
\hline
\end{tabular}

Médias de variedades seguidas de mesma letra minúscula, dentro de cada concentração de fósforo e de alumínio, e médias de concentrações de fósforo, seguidas de mesma letra maiúscula, dentro de cada variedade e concentração de alumínio, não diferem pelo teste $F(P<0,05)$.

quando as variedades foram cultivadas na presença de alumínio e com 32,3 $\mu \mathrm{mol} \mathrm{L}^{-1}$ de fósforo, não havendo diferenças nos demais tratamentos. A eficiência no aproveitamento de fósforo pelas variedades de trigo, considerando o seu suprimento às raízes, está mais condicionada à eficiência de absorção e translocação do que à eficiência de utilização. Rosa \& Camargo (1990) também obtiveram maior matéria seca na Toropi, em relação à CNT 8, em solo com teores tóxicos de alumínio.

\section{CONCLUSÕES}

1. As raízes da variedade de trigo Toropi foram menos afetadas do que as da CNT 8 pelo alumínio; no entanto, não houve relação da absor ção de fósforo com o comprimento, raio médio e superfície do sistema radicular.

2. A presença de alumínio aumentou a diferença entre as variedades Toropi e CNT 8 quanto à eficiência de absorção, transl ocação e utilização de fósforo, com vantagem para a primeira, que é mais tolerante ao alumínio.

\section{AGRADECIMENTOS}

Os autores agradecem ao Prof. Luiz Carlos Federizzi, do Departamento de Plantas de Lavoura da Faculdade de Agronomia da UFRGS, pela orientação na escol ha e fornecimento das sementes das variedades de trigo. 


\section{LITE RATURA CITADA}

ABICHEQUER, A.D \& BOHNEN, H. Eficiência de absorção, transl ocação e utilização de fósforo por variedades de trigo. R. Bras. Ci. Solo, 22:21-26, 1998.

ALVES, V.M.C.; NOVAIS, R.F.; NEVES, J .C.L. \& BARROS, N.F . Efeito do alumínio sobre a absorção etransl ocação de fósforo e sobre a composição mineral de duas cultivares de trigo. Pesq. Agr. Bras., 23:563-573, 1988.

BALIGAR, V.C. \& FAGERIA, N.K. Plant nutrient efficiency: towards the second paradigm. In: SIQUEIRA, J.O.; MOREIRA, F.M.S.; LOPES, A.S.; GUILHERME, L.R.G.; FAQUIN, V.; FURTINI NETO, A.E. \& CARVALHO, J.G., eds. Inter-relação fertilidade, biol ogia do solo e nutrição de plantas. Viçosa: SBCS, Lavras: UFLA/DCS, 1999. 819p.

BEN, J .R. Resposta diferencial de genótipos de trigo (Triticum aestivum L.) a fósforo no solo. Piracicaba, Universidade de São Paulo, 1989. 109p. (Tese de Doutorado)

CANÇADO, G.M.A.; LOPES, M.A. \& PAIVA, E. Genética e bioquímica da tolerância das plantas ao alumínio. In: SIQUEIRA, J.O.; MOREIRA, F.M.S.; LOPES, A.S.; GUILHERME, L.R.G.; FAQUIN, V.; FURTINI NETO, A.E. \& CARVALHO,J.G., eds. Inter-relação fertilidade, biologia do sol o enutrição de plantas. Viçosa: SBCS, Lavras: UFLA/ DCS, 1999. 819p.

CAMARGO, C.E.O.; FELÍCIO, J.C. \& ROCHA JÚNIOR, L.S. Trigo: tolerância ao alumínio em solução nutritiva. Bragantia, 46:183-190, 1987.

CLARKSON, D.T. Effect of aluminum on the uptake and on metabolism of phosphorus by barley seedlings. Plant Physiol., 41:165-172, 1966.

FOY, C.D. Differential aluminum and manganese tolerances of plant species and varieties in acid soils. Ci. Cult., 28:150155, 1976.

FURLANI, P.R. \& FURLANI, A.M.C. Tolerância a alumínio e eficiência a fósforo em milho e arroz: características independentes. Bragantia, 50:331-340, 1991.
GERLOFF, G.C. \& GABELMAN, W.H. Genetic basis of inorganic plant nutrition. In: LÄUCHLI, A. \& BIELESKI, R.L., eds. Inorganic plant nutrition. New York, Springer-Verlag, 1983. p.453-480.

LONERAGAN, J.F. \& ASHER, C.J . Response of plants to phosphate concentration in solution culture: II. Rate of phosphate absorption and its relation to growth. Soil Sci., 103:311-318, 1967.

MARTINEZ, H.E.P.; NOVAIS, R.F.; SACRAMENTO, L.V.S. \& RODRIGUES, L.A. Comportamento de variedades de soja cultivadas sob diferentes concentrações de fósforo: II. Translocação do fósforo absorvido e eficiência nutricional. R. Bras. Ci. Solo, 17:239-244, 1993.

MIRANDA, L.N. Aluminum-phosphate interactions in relation to wheat growth. Reading, University of Reading, 1985. 169p. (Tese de Doutorado)

MURPHY, J . \& RILEY, J .P. A modified single solution method for the determination of phosphatein natural waters. Anal. Chem. Acta, 27:31-36, 1962

ROSA, O.S. \& CAMARGO, C.E.O. Wheat breeding for better efficiency in phosphorus use. In: SAUNDERS, D.A., ed. Wheat for the nontraditional warm areas: a proceeding of the international conference. F oz dol guaçu, U nited Nations Development Programme International Maize and Wheat Improvement Center, 1990. p.333-351.

SHENK, M.K. \& BARBER, S.A. Root characteristics of corn genotypes as related to P uptake. Agr. J ., 48:921-924, 1979.

SIDDIQI, M.Y. \& GLASS, A.D.M. Utilization index: a modified approach to the estimation and comparasion of nutrient utilization efficiency in plants. J. Plant Nutr., 4:289-302, 1981.

SOON, Y.K. Differential response of wheat genotypes to phosphorus in acid soils. J . Plant Nutr., 15:513-526, 1992.

TENNANT, D. A test of a modified line intersect method of estimating root lenght. J . Ecol., 63:995-1000, 1975.

VILELA, L. \& ANGHINONI, I. Morfologia do sistema radicular e cinética da absorção de fósforo em cultivares de soja afetadas pela interação alumínio-fósforo. R. Bras. Ci. Solo, 8:91-96, 1984. 